\title{
Overexpression of TIMP3 inhibits discogenic pain by suppressing angiogenesis and the expression of substance $P$ in nucleus pulposus
}

\author{
MINGWEI HE ${ }^{1 *}$, JINLEI PANG $^{1 *}$, HAIYAN SUN ${ }^{1}$, GUANRONG ZHENG $^{2}$, YAN LIN ${ }^{2}$ and WEIPENG GE $^{2}$ \\ ${ }^{1}$ Department of Anesthesiology, Beijing Anzhen Hospital, Capital Medical University, Beijing 100029; \\ ${ }^{2}$ Department of Pain, Shengli Oilfield Central Hospital, Dongying, Shandong 257000, P.R. China
}

Received January 30, 2019; Accepted September 4, 2019

DOI: $10.3892 / \mathrm{mmr} .2020 .10922$

\begin{abstract}
Approximately 50\% of the cases of low back pain (LBP) are attributed to discogenic origin. The causes of discogenic pain are complicated and consist of a complex biochemical cascade. Neovascularization of intervertebral discs (IVDs) is believed to be associated with discogenic pain. The anti-angiogenesis ability of tissue inhibitor of metalloproteinase-3 (TIMP3) has been reported in many tumors, yet whether TIMP3 is associated with neovascularization of IVDs remains unknown. In the present study, both in vitro and in vivo models were used to investigate the association between discogenic pain and TIMP3 expression in nucleus pulposus (NP). PCR results demonstrated that inflammation induced downregulation of TIMP3 expression in NP cells. By using an adenovirus system to upregulate TIMP3 expression, the effect of TIMP3 on angiogenesis was measured by endothelial cell migration and tube formation assays. The results demonstrated that overexpression of TIMP3 suppressed angiogenesis in NP without the regulation of vascular endothelial growth factor (VEGF) expression. TNF- $\alpha$ converting enzyme (TACE) expression was downregulated by TIMP3, thus inhibiting the TACE-induced activation of TNF- $\alpha$ in NP cells. Immunohistochemical staining of IVDs also confirmed that TIMP3 inhibited the expression of substance P in NP. Taken together, the present results indicated the expression of TIMP3 in NP may have a key role in the development of discogenic pain.
\end{abstract}

Correspondence to: $\mathrm{Dr}$ Weipeng Ge, Department of Pain, Shengli Oilfield Central Hospital, 31 Jinan Road, Dongying, Shandong 257000, P.R. China

E-mail: geweipengs@163.com

*Contributed equally

Key words: tissue inhibitor of metalloproteinase-3, discogenic pain, nucleus pulposus, angiogenesis, TNF- $\alpha$ converting enzyme

\section{Introduction}

Low back pain (LBP) is one of the most common complaints worldwide (1). Approximately 70 to $85 \%$ of the Western population will develop LBP at least once during their lifetime (2). It has been reported that 40 to $50 \%$ of cases of LBP are attributed to discogenic origin $(3,4)$.

The mechanisms of discogenic pain are complicated, consisting of a complex biochemical cascade in which proinflammatory cytokines such as interleukin (IL)- $1 \beta$ and tissue necrosis factor (TNF)- $\alpha$ play an important role (5). Cytokines induce discogenic pain by promoting the expression of pain mediators and inducing inflammatory cascades (6). The upregulation of TNF- $\alpha$ in intervertebral discs (IVDs) may not only contribute to catabolic process, but also be related with discogenic pain $(7,8)$, especially following neural and vascular ingrowth $(9,10)$. Tissue inhibitor of metalloproteinase-3 (TIMP3) was reported to be a suppressor of TNF- $\alpha$ which induces inflammation in various tissues and organs $(11,12)$. However, further studies are needed to clarify the association between TIMP3 and discogenic pain.

Healthy IVDs are aneural and avascular, while neural and vascular ingrowth has been frequently found in degenerated IVDs $(9,10)$, and the neovascularization of IVDs is believed to be associated with discogenic pain (13). Nucleus pulposus (NP) cells were found to induce endothelial cell (EC) invasion by expressing vascular endothelial growth factor (VEGF), especially under inflammatory condition (13). ECs further express nerve growth factor (NGF) that accompany ingrowing nerves (13) and the release of pain mediators (10). VEGF expression in NP cells could be induced by degeneration or inflammation $(13,14)$. TIMP3 is an angiogenesis inhibitor, and the anti-angiogenesis ability of TIMP 3 has been demonstrated in many types of tumor tissues (15-17). However, whether TIMP3 is associated with the neovascularization of IVDs remains unknown.

Substance P (SP) is a sensory marker related to pain. SP-positive nerve fibers were demonstrated to be related to discogenic pain (18). SP expressed in small nociceptive dorsal root ganglion (DRG) neurons is believed to be the sensory transmitter of nociceptive information (19). Numerous studies have demonstrated an association between the expression of $\mathrm{SP}$ in ingrowth nerves and the extent of disc degeneration (13). SP expression is important for pain transmission from the IVD 
and occurrence of discogenic pain. Considering the potential anti-inflammatory and anti-angiogenesis abilities of TIMP3, the relationship between TIMP3 expression and SP release should be further investigated.

Previous studies indicated that TIMP3 expression decreases with age and IVD degeneration (20-23). In addition, previous research has confirmed that this unbalanced expression of TIMP3 may lead to intervertebral disc degeneration (IDD) (24). Despite the finding that the loss of TIMP3 expression leads to IDD, whether TIMP3 expression is related with discogenic pain still lacks direct evidence. In the present study, we explored the relationship between TIMP3 expression and discogenic pain using in vitro and in vivo models.

\section{Materials and methods}

Reagents. The antibodies and reagents used in the present study are as follows: Rat Vascular Endothelial Growth Factor-164 (rVEGF $_{164}$; cat. no. 5874, Cell Signaling Technology, Inc., Danvers, MA, USA), fetal bovine serum (FBS; Gibco, Thermo Fisher Scientific, Inc., Waltham, MA, USA) and lipopolysaccharides (LPS; L5543, Sigma-Aldrich, Merck KGaA, Darmstadt, Germany). Primary antibodies against TIMP3 (ab155749, Abcam, Cambridge, UK), collagen-2 (ab34712, Abcam), GAPDH (ab181603, Abcam), Substance P (ab14184, Abcam), aggrecan (ab36861, Abcam) and CD34 (50589-R013, Sino Biological, Beijing, China) were used in the study. Secondary antibodies for western blotting (ab205718, Abcam) and immunohistochemical analysis (ab205719, Abcam) were also used in the study.

Cell culture. According to previously reported methods, primary nucleus pulposus (NP) cells and rat aorta endothelial cells (RAECs) were isolated from Sprague-Dawley (SD) rats $(24,25)$. A total of $34 \mathrm{SD}$ rats were used for the present study. The SD rats (6 weeks of age) were euthanized using an abdominal injection of pentobarbital sodium $(150 \mathrm{mg} / \mathrm{kg})$. Briefly, NP cells were isolated from lumbar spines and cultured in complete media (high-glucose DMEM with 10\% FBS and $1 \%$ antibiotic). RAECs were isolated from aortas of SD rats and cultured with DMEM/F12 media (with 10\% FBS and 1\% antibiotic). The primary cell procurement and animal experiments were approved by the Animal Experimental Ethics Committee of the Beijing Anzhen Hospital (approval no. 20170614).

Adenovirus vector transfection. Adenovirus vectors loading the coding sequences of rat TIMP3 (NM_012886) or a scramble control were purchased from Sino Biological (Beijing, China). Vectors were amplified on 293 cells (American Type Culture Collection, Manassas, VA. USA), purified, titered and then the particle concentration was measured by optical absorbance. NP cells were transfected with adenovirus vector (TIMP3) or a scrambled control at 50 multiplicity of infection (MOI) according to standard procedure. The transfection efficacy was verified by western blotting 3 days after transfection.

Endothelial cell migration and tubeformationassays. Different $\mathrm{NP}$ cells were cultured for $48 \mathrm{~h}$ and the medium was isolated as conditioned medium (26). For tube formation assays, RAECs were seeding at a density of $1 \times 10^{4} /$ well in 96 -well plates
Table I. Sequences of the primers used in PCR.

\begin{tabular}{lll}
\hline Gene & Primer & \multicolumn{1}{c}{ Sequence $\left(5^{\prime}-3^{\prime}\right)$} \\
\hline TACE & Forward & CCGAACGAGTTTACGGGGAT \\
& Reverse & TGTGCGTCGCCTAGAACTAC \\
TIMP3 & Forward & ACAGACGCCAGAGTCTCCTA \\
& Reverse & ACCTCAAGTCTGTCCGGGTA \\
Substance P & Forward & TTCATCTCCATCTGTGTCCGC \\
& Reverse & GTCTGAGGAGGTCACCACATT \\
TNF- $\alpha$ & Forward & TCGTAGCAAACCACCAAGCA \\
& Reverse & TCGTAGCAAACCACCAAGCA \\
GAPDH & Forward & AACCTTCTTGCAGCTCCTCCG \\
& Reverse & CCATACCCACCATCACACCCT
\end{tabular}

TACE, TNF- $\alpha$ converting enzyme; TIMP3, tissue inhibitor of metalloproteinase- 3 ; TNF- $\alpha$, tissue necrosis factor- $\alpha$.

precoated with Matrigel (356234, BD Biosciences, Franklin Lakes, NJ, USA), and then incubated with different reagents (100 ng/ml VEGF, NP-TIMP3 or NP conditioned medium) for $6 \mathrm{~h}$ according to the different groupings. For cell migration assays, $1 \times 10^{5}$ RAECs were seeding on a Matrigel-coated polycarbonate membrane insert (8.0- $\mu \mathrm{m}$ pores) in a Transwell apparatus (Costar, Corning, NY, USA). Different NP cells (NP and NP-TIMP3) were also cultured with or without $100 \mathrm{ng} / \mathrm{ml}$ VEGF in the lower chamber for $24 \mathrm{~h}$. The cells on the bottom surface of the insert were fixed with $4 \%$ paraformaldehyde and stained with $0.1 \%$ crystal violet. Then the stained cells were observed and counted using a microscope. The formation of tube-like structures and migrated cells were observed under a light microscope (x40 magnification, Olympus). Complete medium without cells was used as the blank control.

Gene expression assay. The total RNA of the various NP cells was isolated using TRIzol reagent (Invitrogen, Thermo Fisher Scientific, Inc.) following the manufacturer's instructions. Reverse transcription was carried out using the 1st Strand cDNA Synthesis Kit (Takara Biotechnology Co., Ltd., Dalian, China). DNA amplification was carried out using the SYBR Premix Ex Taq kit (Takara) followed by real-time PCR. The primers were designed and synthesized by GenePharma (Shanghai, China). Gene expression was measured using the $2^{-\Delta \Delta \mathrm{Cq}}$ method (27). The primer sequences are summarized in Table I, and GAPDH was selected as a reference gene.

Western blot analysis. Aggrecan, Col-2 and TIMP3 protein expression levels were assessed by western blot analysis in NP cells treated with lipopolysaccharide $(1 \mu \mathrm{g} / \mathrm{ml})$ for 2 or 5 days. Briefly, for cell samples, cells were lysed with RIPA buffer and total proteins were isolated. For tissue samples, a tissue homogenate was made and total proteins were isolated. A total of $20 \mu \mathrm{g}$ of each protein sample was separated by $10 \%$ SDS-PAGE, then transferred onto PVDF membranes (EMD Millipore, Billerica, MA, USA). The membranes were blocked with $5 \%$ fat-free milk at room temperature for $1 \mathrm{~h}$, incubated with primary antibodies $(1: 1,000)$ at $4^{\circ} \mathrm{C}$ overnight, followed by secondary antibodies $(1: 5,000)$ at room temperature for 

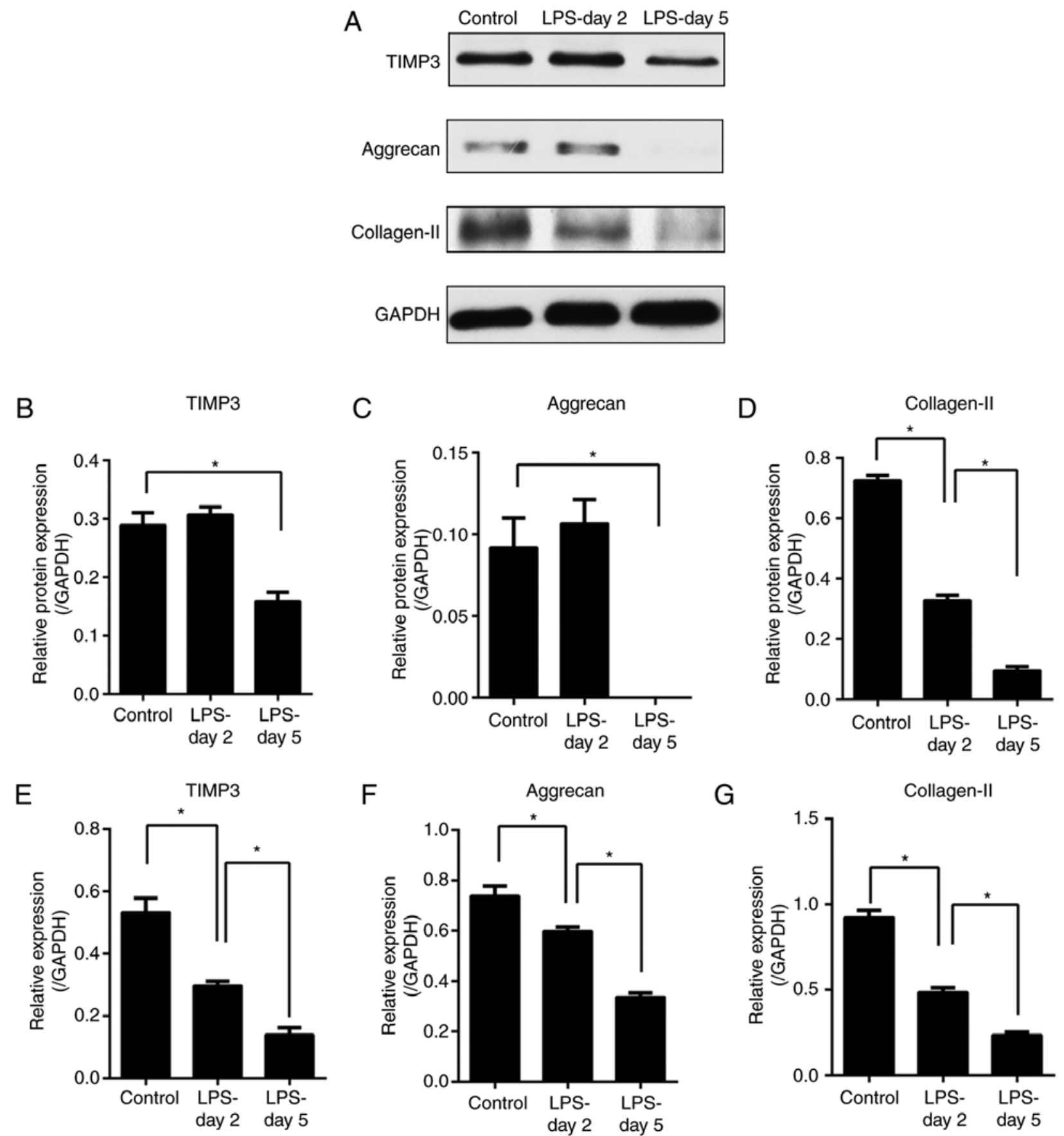

Figure 1. TIMP3 and matrix-related protein expression after LPS stimulation. NP cells were treated with $1 \mu \mathrm{g} / \mathrm{ml}$ LPS for 2 or 5 days. The gene and protein expression of TIMP3, aggrecan and collagen-II was assessed by PCR and western blot analysis. (A-D) Protein levels of TIMP3, aggrecan and collagen-II were measured by western blot analysis. TIMP3 and aggrecan protein expression was downregulated from day 5 , while collagen-II expression was reduced from day 2. (E-G) The gene expression of TIMP3, aggrecan and collagen-II was assessed by PCR. Expression of all genes was downregulated from day 2. Data are presented as the mean \pm SD. ${ }^{*} \mathrm{P}<0.05$. TIMP3, tissue inhibitor of metalloproteinase-3; LPS, lipopolysaccharides.

$1 \mathrm{~h}$. Immunoreactive bands were detected using the Odyssey infrared imaging system (LI-COR). Densitometrical analysis of the bands was completed using Image-Pro Plus 6.0 software supplied by Media Cybernetics, Inc. GAPDH was used as the internal reference.

ELISA assessments. Various NP cells were exposed to LPS $(1 \mu \mathrm{g} / \mathrm{ml})$ for 5 days. The TNF- $\alpha$ level in the culture medium was measured using commercially available enzyme-linked immunosorbent assay (ELISA) kits according to the manu- facturer's instructions (R\&D Systems, Inc., Minneapolis, MN, USA).

Animal experiments. Twenty-four male SD rats (6-8 weeks weighing 200-250 g), were purchased from the Experimental Animal Center of Beijing Anzhen Hospital. Rats were housed under controlled conditions, including a 12-h light/dark cycle, $21 \pm 2^{\circ} \mathrm{C}$ and $60-70 \%$ humidity, and rats were also allowed free access to standard dry rat diet and tap water. The surgical procedure was performed as previously described (28). 
A
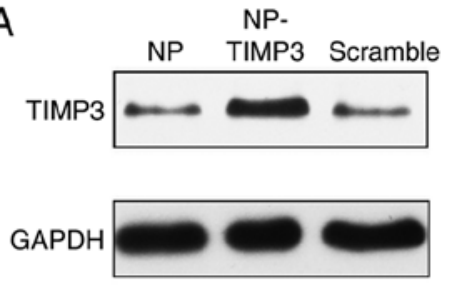

B

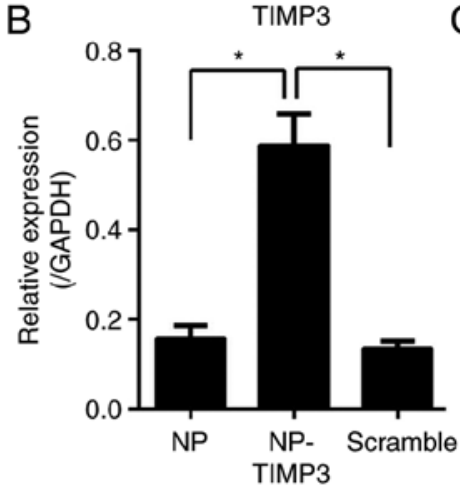

E

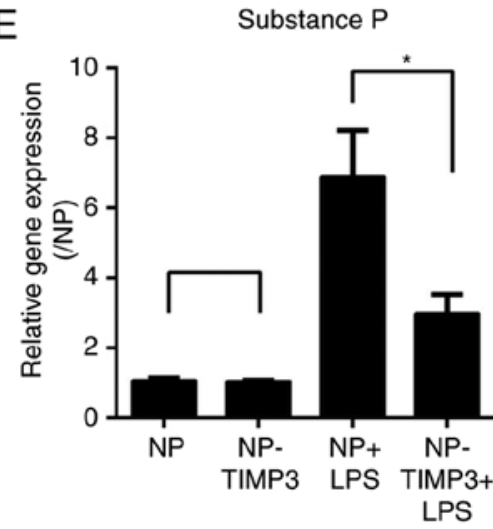

$\mathrm{C}$

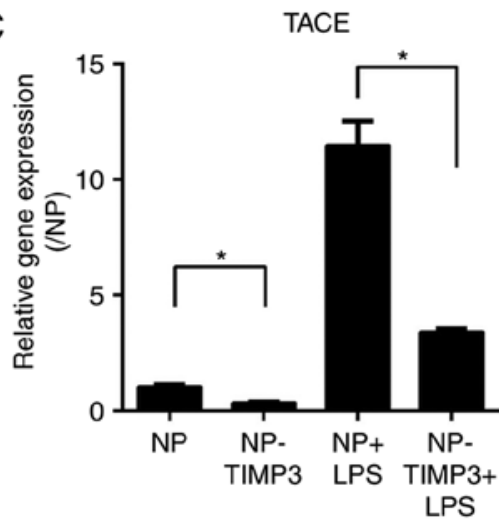

$\mathrm{F}$

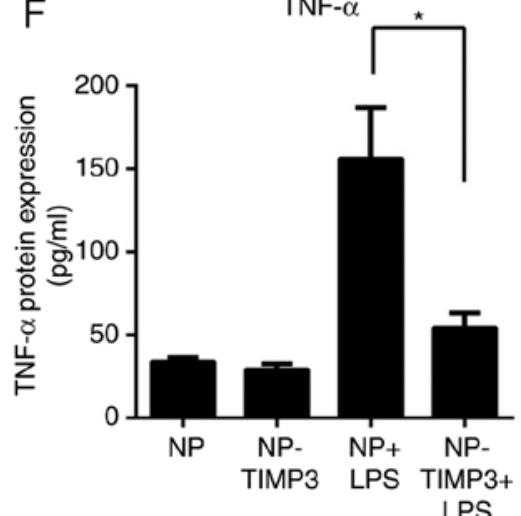

Figure 2. TIMP3 inhibits TNF- $\alpha$ and substance P expression in NP cells. NP cells were transfected with an adenovirus vector loading TIMP3 or a control. The different NP cells were then treated with or without LPS for 2 days. After that, the gene expression of TACE and substance P was determined by PCR. TNF- $\alpha$ secretion was measured by ELISA. (A and B) Overexpression of TIMP3 was confirmed by western blot analysis. (C) Gene expression of TACE was downregulated by TIMP3. (D) Gene expression of TNF- $\alpha$ was not affected by TIMP3. (E) Gene expression of substance P was downregulated by TIMP3. (F) TNF- $\alpha$ secretion was reduced by TIMP3. The data are presented as the mean \pm SD. ${ }^{*} \mathrm{P}<0.05$. TIMP3, tissue inhibitor of metalloproteinase- 3 ; TNF- $\alpha$, tissue necrosis factor- $\alpha$; NP, nucleus pulposus; TACE, TNF- $\alpha$ converting enzyme; LPS, lipopolysaccharides.

Briefly, rats were anesthetized by an abdominal injection of pentobarbital sodium $(40 \mathrm{mg} / \mathrm{kg})$, and percutaneously punctured with a $21 \mathrm{G}$ needle in the coccygeal vertebra (puncture and TIMP3+puncture group). For the TIMP3+puncture group, rats were injected with adenovirus vector $\left(1 \times 10^{9} \mathrm{pfu} / \mathrm{level}\right)$ immediately after puncture. At day 28 after puncture, rats were euthanized by intraperitoneal injection of an overdose of pentobarbital sodium $(150 \mathrm{mg} / \mathrm{kg})$ and nucleus pulposus tissues were isolated for western blot assay and histologic analysis.

Histologic analysis. Discs from rats were fixed and serial sagittal sections of discs (5- $\mu \mathrm{m}$ thick) were obtained to prepare slides. TIMP3, CD34 and substance P expression levels were determined by immunohistochemical (IHC) staining. All staining procedures were performed following standard histochemical protocols. We analyzed 3 sections from each disc samples, and for each section, we calculated 3 fields and took the average. The positive staining was calculated and analyzed using Image-Pro Plus 6.0 software supplied by Media Cybernetics, Inc.

Statistical analysis. All the experiments were repeated at least 3 times. The data are expressed as the mean \pm SD. Statistical analysis was performed with a one-way analysis of variance (ANOVA), followed by Duncan's post hoc test using SPSS
22.0 software (IBM, Inc.). A P-value $<0.05$ was considered to indicate a statistically significant result.

\section{Results}

LPS induces TIMP3, aggrecan and collagen-II downregulation in the NP cells. To study the regulation of TIMP3 expression and the matrix degradation in the presence of inflammation, NP cells were treated with or without LPS for 2 or 5 days. The gene and protein expression of TIMP3, aggrecan and collagen-2 were assessed by PCR (Fig. 1E-G) and western blot analysis (Fig. 1A-D). Our results revealed that gene expression of TIMP3, aggrecan and collagen- 2 were downregulated from day 2 after exposure to LPS (Fig. 1E-G), while significant protein expression reduction was observed only at day 5 except for collagen-II (Fig. 1A-D). These results suggest that the downregulation of TIMP3 plays a role in the matrix degradation of NP cells.

TIMP3 regulates TNF- $\alpha$ and pain mediator expression in NP cells. Considering that the expression of TIMP3 was found to be downregulated after exposure to LPS, we aimed to increase TIMP3 expression in an inflammatory condition and explore the role of TIMP3 in inflammation. TIMP3 was overexpressed by transfection of an adenovirus vector in NP cells. TIMP3 overexpression was confirmed by western blot 
A
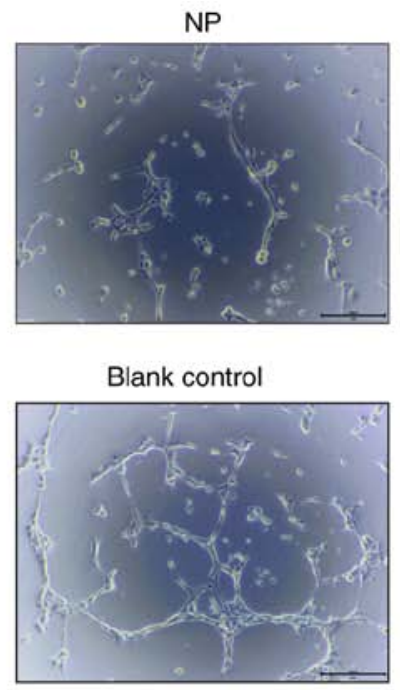

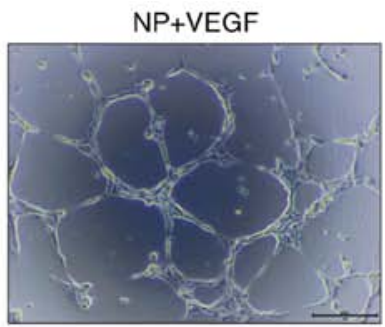

B

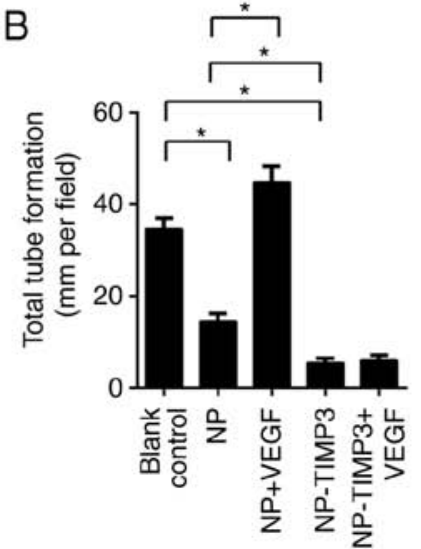

NP-TIMP3

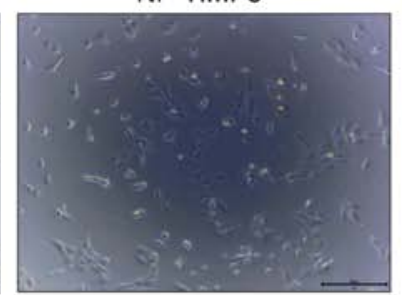

C

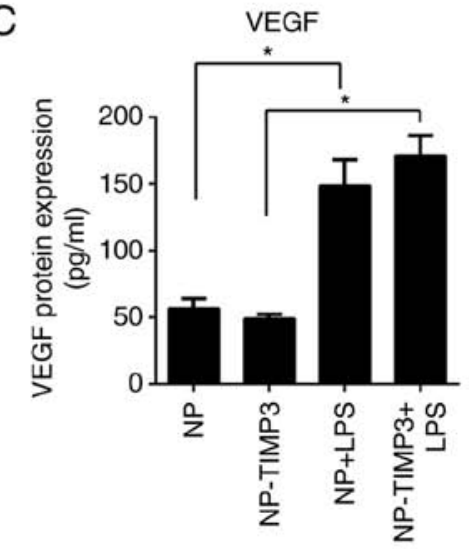

Figure 3. Tube formation and VEGF secretion measurement. RAECs were seeding at a density of $1 \times 10^{4} /$ well in 96 -well plates precoated with Matrigel, and then incubated with different reagents (100 ng/ml VEGF, NP-TIMP3 or NP conditioned medium) for $6 \mathrm{~h}$ according to the different groupings. Tube formation of RAECs was observed and analyzed under a light microscope. VEGF secretion by NP cells was also assessed after treatment with $1 \mu \mathrm{g} / \mathrm{ml} \mathrm{LPS}$ or not. (A and B) Tube formation assay of RAECs. NP-TIMP3 significantly reduced the tube formation of RAECs with or without VEGF stimulation. (C) ELISA assay of VEGF secretion by NP cells. LPS induced VEGF secretion in NP cells. Data are presented as the mean \pm SD. "P<0.05. Scale bar, $20 \mu \mathrm{m}$. VEGF, vascular endothelial growth factor; RAECs, rat aorta endothelial cells; TIMP3, tissue inhibitor of metalloproteinase-3; NP, nucleus pulposus; LPS, lipopolysaccharides.

A

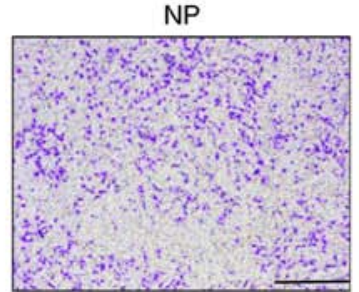

NP-TIMP3+VEGF

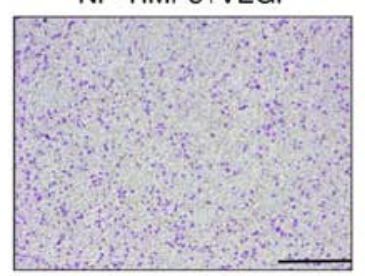

NP+VEGF

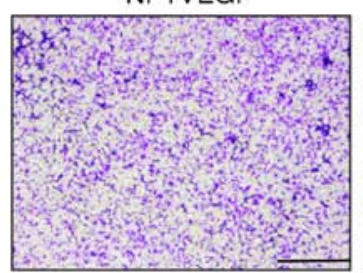

Blank control

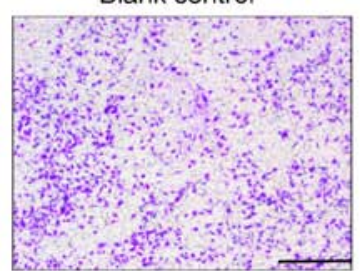

NP-TIMP3
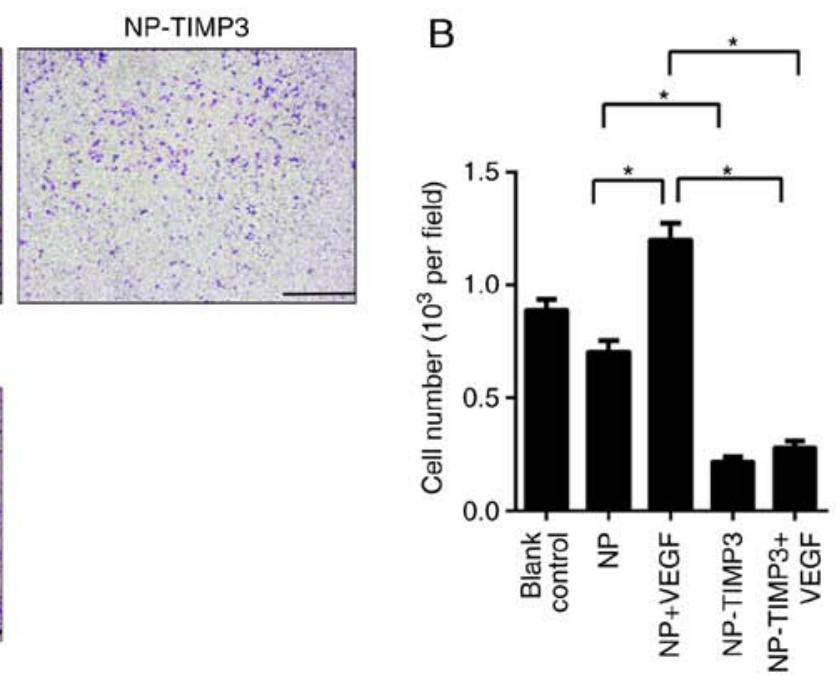

Figure 4. Endothelial cell migration assay. RAECs $\left(1 \times 10^{5}\right)$ were seeding on a Matrigel-coated polycarbonate membrane insert in a Transwell apparatus. Different NP cells (NP and NP-TIMP3) were also cultured with or without $100 \mathrm{ng} / \mathrm{ml}$ VEGF in the lower chamber for $24 \mathrm{~h}$. For the blank control group, only medium was added into the lower chamber. The cells on the bottom surface of the insert were fixed and staining. Then the stained cells were observed and counted by a microscope. (A and B) RAEC migration was measured by Transwell assay. VEGF significantly increased the RAEC migration in the NP and NP-TIMP3 groups, while NP-TIMP3 significantly reduced migration of RAECs with or without VEGF stimulation. Data are presented as the mean \pm SD. ${ }^{*} \mathrm{P}<0.05$. Scale bar, $100 \mu \mathrm{m}$. RAECs, rat aorta endothelial cells; NP, nucleus pulposus; TIMP3, tissue inhibitor of metalloproteinase-3; VEGF, vascular endothelial growth factor.

analysis (Fig. 2A and B). After exposure to LPS, the gene expression of TNF- $\alpha$ converting enzyme (TACE), TNF- $\alpha$ and substance $\mathrm{P}$ in different NP cells was measured by qPCR. The results showed that TIMP3 overexpression downregulated TACE and substance $\mathrm{P}$ expression which were induced by
LPS (Fig. 2C and E). Although the gene expression of TNF- $\alpha$ was not affected upon overexpression of TIMP3 (Fig. 2D), the ELISA measurement indicated that the protein level of TNF- $\alpha$ in medium was decreased in the TIMP3+LPS group (Fig. 2F). Taken together, our results suggest that instead of 
A

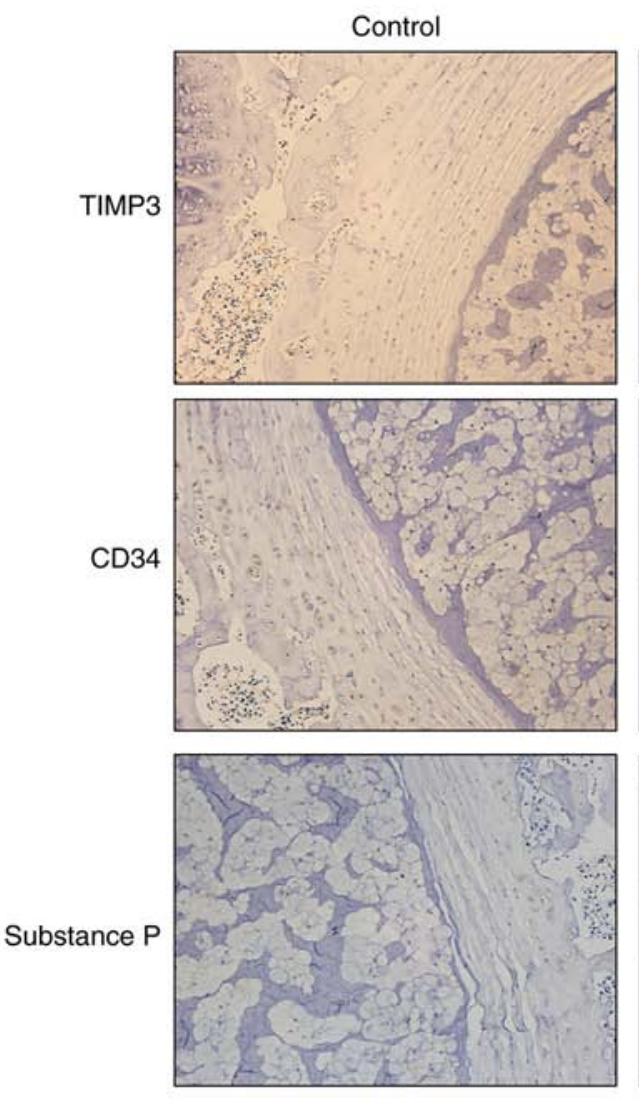

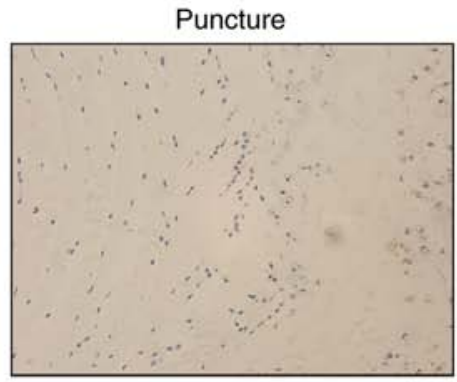
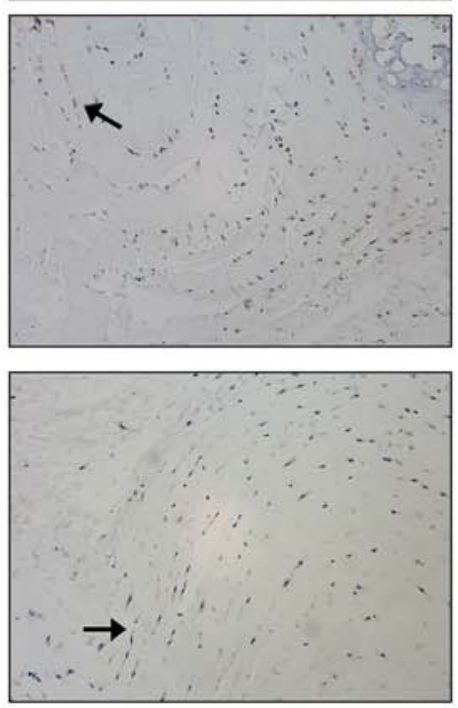
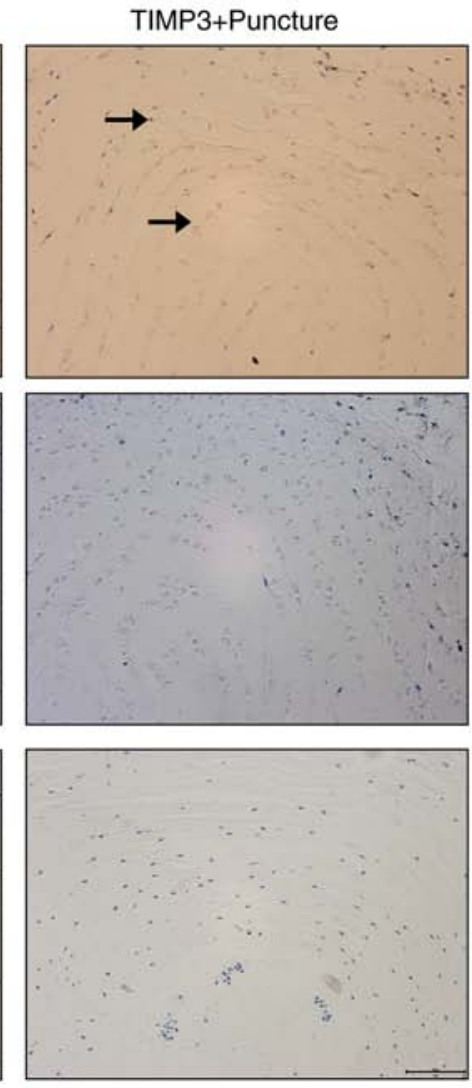

B

Control

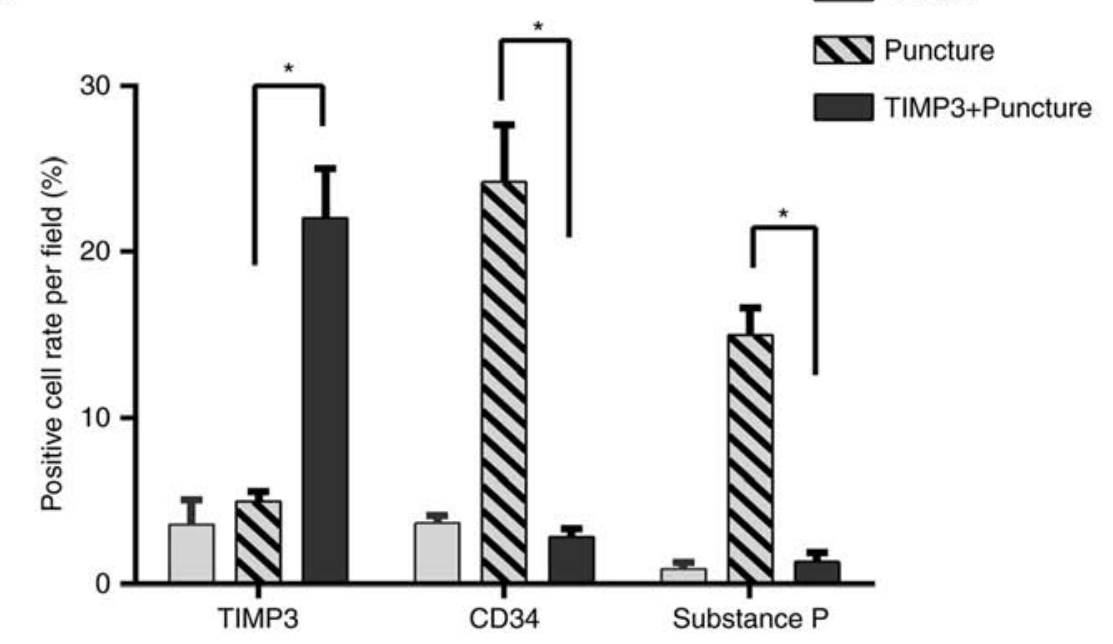

Figure 5. TIMP3, CD34 and substance P expression in rat NP tissue. Rats were percutaneously punctured with a 21G needle in coccygeal vertebra (puncture and TIMP3+puncture group). For TIMP3+puncture group, rats were injected with adenovirus vector (1x109 pfu/level) immediately after puncture. At day 28 after puncture, nucleus pulposus (NP) tissues were isolated for immunohistochemical staining. (A and B) Immunohistochemical staining was performed to measure TIMP3, CD34 and substance P expression in rat NP tissue. Overexpression of TIMP3 could reduce CD34 and substance P expression in NP tissue compared with the puncture group. Black arrow, positive staining. Data are presented as the mean $\pm \mathrm{SD}$. " $\mathrm{P}<0.05$. Scale bar, $20 \mu \mathrm{m}$. TIMP3, tissue inhibitor of metalloproteinase-3.

directly inhibiting TNF- $\alpha$ expression, TIMP3 was more likely to suppress the activation of TNF- $\alpha$ induced by TACE.

TIMP3 inhibits tube formation and migration of RAECs. According to ELISA measurement, NP cells secreted VEGF after stimulation with LPS (Fig. 3C). To further investigate the association between angiogenesis and TIMP3, RAECs were treated with different conditioned medium and VEGF or not. Tube formation was observed under a light microscope. As shown in Fig. 3, NP cell medium significantly suppressed tube formation of RAECs, while overexpression of TIMP3 further significantly inhibited tube formation. Treatment with VEGF promoted tube formation of RAECs, however, this promoting effect was blocking by overexpression of TIMP3 (Fig. 3). The results of the cell migration assay were similar to that of the tube formation assay (Fig. 4). TIMP3 significantly 


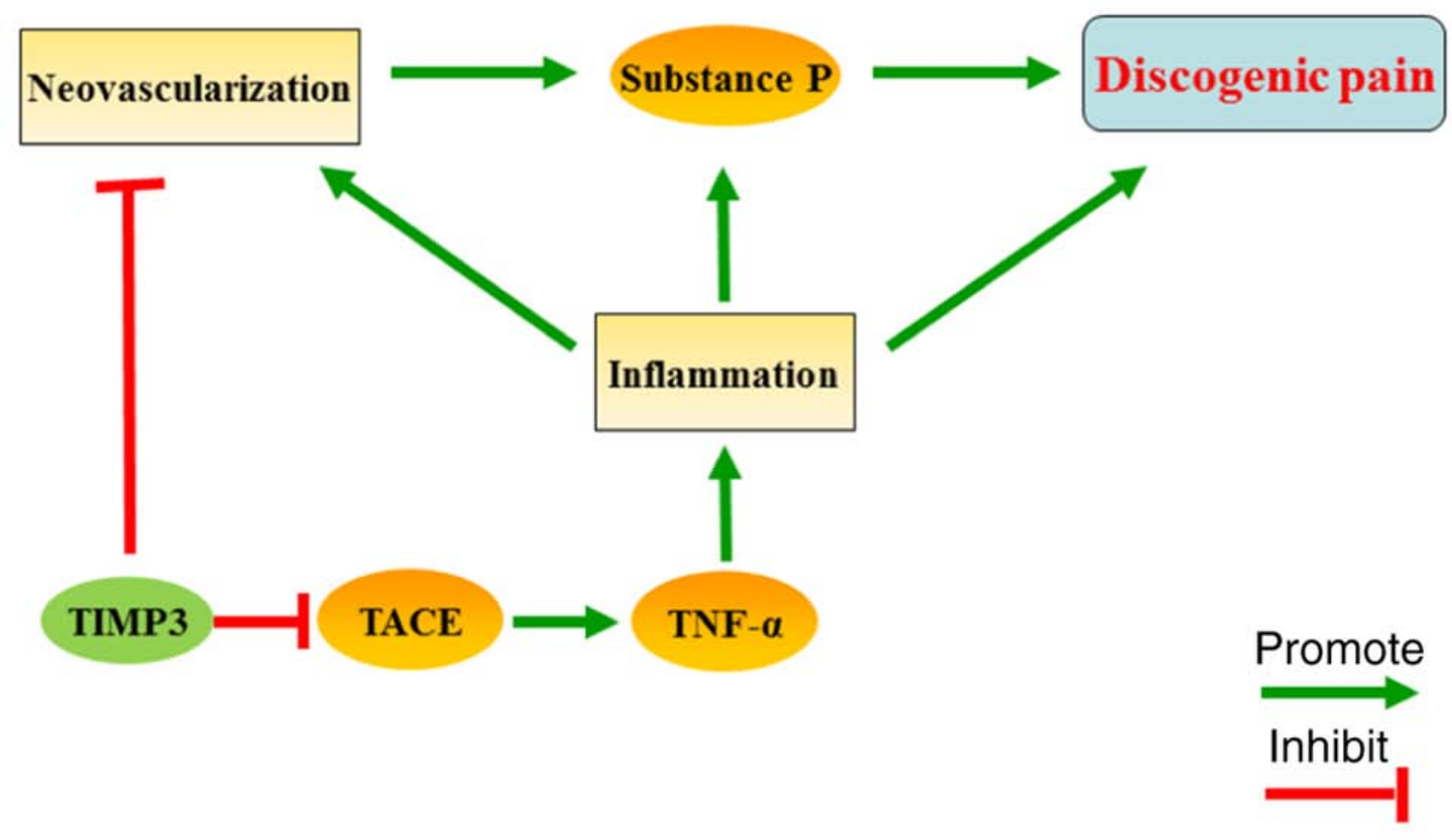

Figure 6. Schematic diagram of the mechanism of TIMP3 in discogenic pain. TIMP3 inhibits discogenic pain by suppressing angiogenesis and the expression of substance P. TIMP3, tissue inhibitor of metalloproteinase- 3 ; TNF- $\alpha$, tissue necrosis factor- $\alpha$; TACE, TNF- $\alpha$ converting enzyme.

inhibited the migration ability of the RAECs and eliminated the promoting effect of VEGF. According to the above results, TIMP3 inhibits angiogenesis without regulating VEGF expression, indicated that TIMP3 may inhibit angiogenesis by blocking the bonding of VEGF and VEGFR-2.

Overexpression of TIMP3 reduces pain mediator expression in IVD. Substance P is a key pain mediator in IVD, and CD34 is a marker of angiopoiesis. Considering the inhibitory effects of TIMP3 on substance P expression and angiopoiesis, these effects were confirmed in an in vitro model. The inhibitory effect of TIMP3 on discogenic pain was further investigated in an in vivo model by assaying substance $\mathrm{P}$ and CD34 expression. IDD rat model was established by puncture of IVD. After injection of an adenovirus vector loading TIMP3, TIMP3 expression was significantly upregulated at day 28 (Fig. 5). The puncture group exhibited more positive CD34 and substance P staining, which indicated the neovascularization of IVDs after puncture. The positive staining rate of CD34 and substance $\mathrm{P}$ was significantly reduced in the TIMP3+puncture group compared with that in the control group (Fig. 5). These results indicate that TIMP3 may suppress the angiogenesis and pain mediator expression in degenerative discs, thus inhibiting discogenic pain in IVD. On the other hand, the loss of TIMP3 expression may play a role in the development of discogenic pain.

\section{Discussion}

As a key matrix-degrading enzyme inhibitor, the imbalance of expression between TIMP3 and matrix-degrading enzymes has been reported to be responsible for the aggrecan breakdown in intervertebral discs (IVDs) (20-22,24). Lipopolysaccharides (LPS) markedly induce inflammation in nucleus pulposus (NP) cells (29). And LPS also induce the gene and protein overexpression of various matrix-degrading enzymes, thus leading to matrix degradation $(20,30)$. In the present study, exposure to LPS induced the downregulation of collagen-II and aggrecan at both the gene and protein levels, and the TIMP3 expression was also inhibited by LPS, which was consistent with a previous study (24). In addition to direct inhibition of enzyme activity, TIMP-3 also provides a feedback gene downregulation of matrix-degrading enzymes (24).

The relationship between TNF- $\alpha$ and discogenic pain has been studied for years. There are two forms of TNF- $\alpha$ in humans, membrane-bound (mTNF- $\alpha$ ) and a secreted form $(\mathrm{sTNF}-\alpha)$. The transformation from mTNF- $\alpha$ to $\mathrm{sTNF}-\alpha$ is processed by TACE. Increased levels of STNF- $\alpha$ were reported to present in degenerate and herniated IVDs $(31,32)$. TNF- $\alpha$ may trigger discogenic pain in several ways, including sensitization of neurons, induction of inflammation and upregulation of various receptors $(33,34)$. These modifications promote the release of pain mediators, resulting in chronic, persistent pain $(33,35)$. Although there is still controversy concerning the clinical effect, the use of anti-TNF- $\alpha$ has been proven effective in discogenic pain (35-38). TIMP3 was reported to be a suppressor of $\mathrm{TNF}-\alpha$-induced inflammation in various tissues and organs $(11,12)$. Our study also showed that instead of directly inhibited TNF- $\alpha$ expression, TIMP3 suppressed TNF- $\alpha$ secretion by downregulating TACE expression. As a previous study reported, intradiscal administration of TNF- $\alpha$ inhibitor could alleviate discogenic pain for up to 8 weeks (39). Based on our results, overexpression of TIMP3 in NP cells may be able to relieve discogenic pain by the inhibition of TNF- $\alpha$.

SP has been reported to be a sensory marker which is released by nerve fiber. However, in an inflammatory pain model, SP release was also increased (40). The interplay 
between inflammatory cytokines and neurotrophins, which is produced by disc cells may explain the phenomenon. Neurotrophins such as NGF expression was found to be increased in painful and degenerate discs and are sensitive to TNF- $\alpha(13,35)$. Neurotrophins would further promote nerve ingrowth and the release of SP $(13,14)$. Collectively, in light of the present results and the research mentioned above, we deduced that TIMP3 may suppress SP released by the inhibition of TNF- $\alpha$ expression. Innervation is limited in the normal disc, while abundance of nociceptive nerve endings was found in patients with chronic back pain $(9,13)$. Neural ingrowth in IVDs was found to be accompanied by angiogenesis, and nerve growth factor (NGF) was expressed by vascular tissue, thus promoting neural ingrowth (13). Moreover, NGF was found to be expressed only in blood vessels in painful degenerative IVDs (13), which further confirms the close relationship between angiogenesis and discogenic pain. VEGF is an important growth factor of angiogenesis. Overexpression of VEGF was found in NP cells under inflammatory or degenerated situations, which was considered to be one of the key reasons causing angiogenesis (14). Co-culture model also showed that Fas ligand (FasL) and immune privilege were involved in the process of IVD angiogenesis, indicating a more complicated mechanism of neovascularization of IVDs $(41,42)$. TIMP3 was reported as an effective angiogenesis inhibitor in various tumor tissues, which suppressed vascular ingrowth by interfering with the binding of VEGF and VEGFR-2 (15). TIMP3 also inhibits the inflammation which was proven as a trigger of VEGF release $(11,12,14)$. Moreover, aggrecan from healthy IVDs inhibited neural and vascular ingrowth (43). Downregulation of TIMP3 may cause matrix degradation, thus breaking the defense effect provided by intact aggrecan leading to discogenic pain. Our study further confirmed the anti-angiogenesis effect of TIMP3 and interruption of the positive feedback of neural ingrowth in IVD. However, the tube formation results seemed a bit confusing. The NP group exhibited suppressed tube formation compared with the blank control. We speculated that this occurred due to the influence of notochord cells. The NP cells were collected from young rat IVDs which consist of a certain amount of notochord cells, and notochord cells were reported to be able to inhibit the vascular ingrowth in IVDs (44). We did not separate these notochord cells from NP cells in our study. The mixed state of NP and notochord cells was more in accord with the natural state of an organism.

Combined with the results above, we believe that TIMP3 may reduce the neovascularization of IVDs and suppress the inflammation-related SP release, and ultimately inhibit discogenic pain (Fig. 6). However, there are still some limitations of our study. First, the detailed mechanism of the anti-angiogenesis effect of TIMP3 was not thoroughly studied. The interaction between VEGF and VEGFR-2 was not demonstrated experimentally. Moreover, all of the conclusions in the present study were based on in vivo and in vitro experiments. More data from clinical samples are needed in the future.

In conclusion, our in vitro and in vivo studies indicate that overexpression of TIMP3 inhibits discogenic pain by suppressing angiogenesis and the expression of pain mediator in nucleus pulposus. TIMP-3 may play an important role in the pathogenesis of discogenic pain and therefore be a potential therapeutic target.

\section{Acknowledgements}

Not applicable.

\section{Funding}

No funding was received.

\section{Availability of data and materials}

The datasets used and/or analyzed during the current study are available from the corresponding author on reasonable request.

\section{Authors' contributions}

MWH, JLP, WPG and GRZ conceived and designed the study. MWH, JLP and HYS performed the experiments. MWH, JLP and HYS wrote the paper. YL and MWH analyzed the data. GRZ, YL and WPG reviewed and edited the manuscript. All authors read and approved the manuscript and agree to be accountable for all aspects of the research in ensuring that the accuracy or integrity of any part of the work are appropriately investigated and resolved.

\section{Ethics approval and consent to participate}

The primary cell procurement and animal experiments were approved by the Animal Experimental Ethics Committee of the Beijing Anzhen Hospital (approval no. 20170614).

\section{Patient consent for publication}

Not applicable.

\section{Competing interests}

The authors declare that they have no competing interests.

\section{References}

1. Vos T, Flaxman AD, Naghavi M, Lozano R, Michaud C, Ezzati M, Shibuya K, Salomon JA, Abdalla S, Aboyans V, et al: Years lived with disability (YLDs) for 1160 sequelae of 289 diseases and injuries 1990-2010: A systematic analysis for the Global Burden of Disease Study 2010. Lancet 380: 2163-2196, 2012.

2. Andersson GB: Epidemiological features of chronic low-back pain. Lancet 354: 581-585, 1999.

3. DePalma MJ,Ketchum JM and Saullo T: What is the source of chronic low back pain and does age play a role? Pain Med 12: 224-233, 2011.

4. Schwarzer AC, Aprill CN, Derby R, Fortin J, Kine G and Bogduk N: The prevalence and clinical features of internal disc disruption in patients with chronic low back pain. Spine (Phila Pa 1976) 20: 1878-1883, 1995.

5. Wuertz K and Haglund L: Inflammatory mediators in intervertebral disk degeneration and discogenic pain. Global Spine J 3: 175-184, 2013.

6. Kepler CK, Ponnappan RK, Tannoury CA, Risbud MV and Anderson DG: The molecular basis of intervertebral disc degeneration. Spine J 13: 318-330, 2013.

7. Bachmeier BE, Nerlich AG, Weiler C, Paesold G, Jochum M and Boos N: Analysis of tissue distribution of TNF-alpha, TNF-alpha-receptors, and the activating TNF-alpha-converting enzyme suggests activation of the TNF-alpha system in the aging intervertebral disc. Ann N Y Acad Sci 1096: 44-54, 2007. 
8. Igarashi T, Kikuchi S, Shubayev V and Myers RR: 2000 Volvo Award winner in basic science studies: Exogenous tumor necrosis factor-alpha mimics nucleus pulposus-induced neuropathology. Molecular, histologic, and behavioral comparisons in rats. Spine (Phila Pa 1976) 25: 2975-2980, 2000.

9. Boos N, Weissbach S, Rohrbach H, Weiler C, Spratt KF and Nerlich AG: Classification of age-related changes in lumbar intervertebral discs: 2002 Volvo Award in basic science. Spine (Phila Pa 1976) 27: 2631-2644, 2002.

10. Freemont AJ, Peacock TE, Goupille P, Hoyland JA, O'Brien J and Jayson MI: Nerve ingrowth into diseased intervertebral disc in chronic back pain. Lancet 350: 178-181, 1997.

11. Smookler DS, Mohammed FF, Kassiri Z, Duncan GS, Mak TW and Khokha R: Tissue inhibitor of metalloproteinase 3 regulates TNF-dependent systemic inflammation. J Immunol 176: 721-725, 2006.

12. Mohammed FF, Smookler DS, Taylor SE, Fingleton B, Kassiri Z, Sanchez OH, English JL, Matrisian LM, Au B, Yeh WC and Khokha R: Abnormal TNF activity in Timp3-/- mice leads to chronic hepatic inflammation and failure of liver regeneration. Nat Genet 36: 969-977, 2004.

13. Freemont AJ, Watkins A, Le Maitre C, Baird P, Jeziorska M, Knight MT, Ross ER, O'Brien JP and Hoyland JA: Nerve growth factor expression and innervation of the painful intervertebral disc. J Pathol 197: 286-292, 2002.

14. Binch AL, Cole AA, Breakwell LM, Michael AL, Chiverton N, Cross AK and Le Maitre CL: Expression and regulation of neurotrophic and angiogenic factors during human intervertebral disc degeneration. Arthritis Res Ther 16: 416, 2014.

15. Janssen A, Hoellenriegel J, Fogarasi M, Schrewe H, Seeliger M, Tamm E, Ohlmann A, May CA, Weber BH and Stöhr H: Abnormal vessel formation in the choroid of mice lacking tissue inhibitor of metalloprotease-3. Invest Ophthalmol Vis Sci 49: 2812-2822, 2008.

16. Das AM, Koljenović S, Oude Ophuis CM, van der Klok T, Galjart B, Nigg AL, van Cappellen WA, Noordhoek Hegt V, Dinjens WN, Atmodimedjo PN, et al: Association of TIMP3 expression with vessel density, macrophage infiltration and prognosis in human malignant melanoma. Eur J Cancer 53: 135-143, 2016.

17. Välimäki J and Uusitalo H: Matrix metalloproteinases (MMP-1, MMP-2, MMP-3 and MMP-9, and TIMP-1, TIMP-2 and TIMP-3) and markers for vascularization in functioning and non-functioning bleb capsules of glaucoma drainage implants. Acta Ophthalmol 93: 450-456, 2015

18. Yamauchi K, Inoue G, Koshi T, Yamashita M, Ito T, Suzuki M, Eguchi Y, Orita S, Takaso M, Nakagawa K, et al: Nerve growth factor of cultured medium extracted from human degenerative nucleus pulposus promotes sensory nerve growth and induces substance P in vitro. Spine (Phila Pa 1976) 34: 2263-2269, 2009.

19. Kuraishi Y, Hirota N, Sato Y, Hino Y, Satoh M and Takagi H: Evidence that substance $\mathrm{P}$ and somatostatin transmit separate information related to pain in the spinal dorsal horn. Brain Res 325: 294-298, 1985

20. Le Maitre CL, Freemont AJ and Hoyland JA: Localization of degradative enzymes and their inhibitors in the degenerate human intervertebral disc. J Pathol 204: 47-54, 2004.

21. Huang $M$, Wang HQ, Zhang Q, Yan XD, Hao M and Luo ZJ Alterations of ADAMTSs and TIMP-3 in human nucleus pulposus cells subjected to compressive load: Implications in the pathogenesis of human intervertebral disc degeneration. J Orthop Res 30: 267-273, 2012.

22. Pockert AJ, Richardson SM, Le Maitre CL, Lyon M, Deakin JA, Buttle DJ, Freemont AJ and Hoyland JA: Modified expression of the ADAMTS enzymes and tissue inhibitor of metalloproteinases 3 during human intervertebral disc degeneration. Arthritis Rheum 60: 482-491, 2009.

23. Tsuji T, Chiba K, Imabayashi H, Fujita Y, Hosogane N, Okada Y and Toyama Y: Age-related changes in expression of tissue inhibitor of metalloproteinases-3 associated with transition from the notochordal nucleus pulposus to the fibrocartilaginous nucleus pulposus in rabbit intervertebral disc. Spine (Phila $\mathrm{Pa}$ 1976) 32: 849-856, 2007

24. Li Y, Li K, Han X, Mao C, Zhang K, Zhao T and Zhao J: The imbalance between TIMP3 and matrix-degrading enzymes plays an important role in intervertebral disc degeneration. Biochem Biophys Res Commun 469: 507-514, 2016.

25. Meng F, Zhao Y, Wang B, Li B, Sheng Y, Liu M, Li H and Xiu R: Endothelial cells promote calcification in aortic smooth muscle cells from spontaneously hypertensive rats. Cell Physiol Biochem 49: 2371-2381, 2018.
26. Fang JH, Zheng ZY, Liu JY, Xie C, Zhang ZJ and Zhuang SM Regulatory role of the MicroRNA-29b-IL-6 signaling in the formation of vascular mimicry. Mol Ther Nucleic Acids 8: 90-100, 2017.

27. Livak KJ and Schmittgen TD: Analysis of relative gene expression data using real-time quantitative PCR and the 2-Delta Delta C(T)) method. Methods 25: 402-408, 2001.

28. Chen T, Cheng X, Wang J, Feng X and Zhang L: Time-course investigation of intervertebral disc degeneration induced by different sizes of needle punctures in rat tail disc. Med Sci Monit 24: 6456-6465, 2018.

29. Kim JS, Ellman MB, Yan D, An HS, Kc R, Li X, Chen D, Xiao G, Cs-Szabo G, Hoskin DW, et al: Lactoferricin mediates anti-inflammatory and anti-catabolic effects via inhibition of IL-1 and LPS activity in the intervertebral disc. J Cell Physiol 228: 1884-1896, 2013

30. Ellman MB, Kim JS, An HS, Chen D, KC R, An J, Dittakavi T, van Wijnen AJ, Cs-Szabo G, Li X, et al: Toll-like receptor adaptor signaling molecule MyD88 on intervertebral disk homeostasis: In vitro, ex vivo studies. Gene 505: 283-290, 2012.

31. Le Maitre CL, Freemont AJ and Hoyland JA: The role of interleukin-1 in the pathogenesis of human intervertebral disc degeneration. Arthritis Res Ther 7: R732-R745, 2005.

32. Le Maitre CL, Hoyland JA amd Freemont AJ: Catabolic cytokine expression in degenerate and herniated human intervertebral discs: IL-1beta and TNFalpha expression profile. Arthritis Res Ther 9: R77, 2007.

33. Schaible HG, Schmelz M and Tegeder I: Pathophysiology and treatment of pain in joint disease. Adv Drug Deliv Rev 58: 323-342, 2006 .

34. Calich AL, Domiciano DS and Fuller R: Osteoarthritis: Can anti-cy tokine therapy play a role in treatment? Clin Rheumatol 29: 451-455, 2010.

35. Schäfers M, Svensson CI, Sommer C and Sorkin LS: Tumor necrosis factor-alpha induces mechanical allodynia after spinal nerve ligation by activation of p38 MAPK in primary sensory neurons. J Neurosci 23: 2517-2521, 2003.

36. Olmarker K, Nutu M and Størkson R: Changes in spontaneous behavior in rats exposed to experimental disc herniation are blocked by selective TNF-alpha inhibition. Spine (Phila Pa 1976) 28: 1635-1641, 2003.

37. Schäfers M and Sommer C: Anticytokine therapy in neuropathic pain management. Expert Rev Neurother 7: 1613-1627, 2007.

38. Olmarker $\mathrm{K}$ and Larsson $\mathrm{K}$ : Tumor necrosis factor alpha and nucleus-pulposus-induced nerve root injury. Spine (Phila $\mathrm{Pa}$ 1976) 23: 2538-2544, 1998

39. Sainoh T, Orita S, Miyagi M, Inoue G, Kamoda H, Ishikawa T, Yamauchi K, Suzuki M, Sakuma Y, Kubota G, et al: Single intradiscal administration of the tumor necrosis factor-alpha inhibitor, etanercept, for patients with discogenic low back pain. Pain Med 17: 40-45, 2016.

40. Noguchi K and Ruda MA: Gene regulation in an ascending nociceptive pathway: Inflammation-induced increase in preprotachykinin mRNA in rat lamina I spinal projection neurons. J Neurosci 12: 2563-2572, 1992.

41. Sun Z, Wan ZY, Guo YS, Wang HQ and Luo ZJ: FasL on human nucleus pulposus cells prevents angiogenesis in the disc by inducing Fas-mediated apoptosis of vascular endothelial cells. Int J Clin Exp Pathol 6: 2376-2385, 2013.

42. Liu ZH, Sun Z, Wang HQ, Ge J, Jiang TS, Chen YF, Ma Y, Wang C, Hu S, Samartzis D and Luo ZJ: FasL expression on human nucleus pulposus cells contributes to the immune privilege of intervertebral disc by interacting with immunocytes. Int J Med Sci 10: 1053-1060, 2013.

43. Johnson WE, Caterson B, Eisenstein SM, Hynds DL, Snow DM and Roberts S: Human intervertebral disc aggrecan inhibits nerve growth in vitro. Arthritis Rheum 46: 2658-2664, 2002.

44. Cornejo MC, Cho SK, Giannarelli C, Iatridis JC and Purmessur D: Soluble factors from the notochordal-rich intervertebral disc inhibit endothelial cell invasion and vessel formation in the presence and absence of pro-inflammatory cytokines. Osteoarthritis Cartilage 23: 487-496, 2015.

This work is licensed under a Creative Commons Attribution-NonCommercial-NoDerivatives 4.0 International (CC BY-NC-ND 4.0) License. 